\title{
Interactive comment on "Feedback of mesoscale ocean currents on atmospheric winds in high-resolution coupled models and implications for the forcing of ocean-only models" by Rafael Abel et al.
}

\section{Anonymous Referee \#1}

Received and published: 15 May 2017

Comments on "Feedback of mesoscale ocean currents on atmospheric winds in high-resolution coupled models and implications for the forcing of ocean-only models" by Abel et al

In this manuscript, the authors aim to test a parameterization of the atmospheric response to so-called current feedback to the atmosphere. Using global coupled model with a spatial resolution of $1 / 4$ and $1 / 12$, they first confirm the existence of linear relationships between surface currents and wind and and surface currents and wind stress. 
The associated coupling coefficients $s_{w}$ and $s_{s t}$ defined in previous studies are then estimated and used 1) to test a parameterization of the wind response to the current feedback in uncoupled ocean model and (2) to validate such a parameterization. This paper addresses relevant scientific questions, an uncoupled oceanic simulation should incorporate the current feedback to the atmosphere but also the atmospheric response through a parameterization. Although the premise of the study is interesting, there are some strong limitations that should be addressed in order to properly test the parameterization and to give some robustness to the conclusions. In particular the model setup of the uncoupled oceanic simulations should be better designed and the results should be better analyzed (see general comments). The results are therefore not sufficient to support the interpretations and conclusions. The introduction should be improved by better acknowledging what is really new in that paper and what has been done before. The results should also be compared to the existing literature. The overall presentation is well structured and clear and the language is fluent enough.

General Comments:

1) I'm seriously worried by the model setup that presents many weaknesses. This should be addressed before trying to test and to validate the parameterization as those weaknesses may have a strong influence on the results. There are many difference between the coupled simulations and the uncoupled ocean model, making their comparison impossible.

a) The atmospheric forcing is drastically different. The CORE forcing has been chosen to force the ocean model. Why don't use the atmospheric forcing from the coupled simulation? the CORE forcing has a very low spatial resolution and a lower temporal frequency (that should be specified). The heat fluxes are certainly very different and the CORE wind would not be comparable to the coupled wind, especially in the nearshore regions. The atmospheric forcing frequency also introduces difference between the coupled run and the uncoupled run. The use of a such a different atmospheric product is problematic because the coupled model is not comparable to the uncoupled model,

OSD

Interactive comment
Printer-friendly version

Discussion paper

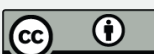


and the estimated coupling coefficients from the coupled model may not be valid for the uncoupled simulation.

OSD

b) The bulk formulae is also very different, this will also introduce difference between the uncoupled and the coupled simulations (see e.g. Brodeau et al. 2016).

c) The spatial resolution of the uncoupled domain is too coarse. A 1/4 ocean model is only eddy permitting. A $1 / 12$ ocean model will not surprisingly generate much more EKE than a 1/4 degree model. This is an important issue when addressing the impact of the current feedback on the EKE and testing the proposed parameterization using among other diagnostics the EKE and the MKE. An eddy resolving model is needed to properly assess the EKE dampening by the current feedback and its partial re-energization by the atmospheric response.

The mean states of the uncoupled models and of the coupled models are likely very different as the EKE should be as recognized by the authors in the paper. The parameterization should be tested on an eddy resolving model. The ocean uncoupled model should be run with a 1/12 spatial resolution using the atmospheric from a coupled simulation with the very same frequency, bulk formulae, etc .. it should try to mimic the coupled simulation. Also, even if the authors refer to another papers, the model setup description should be improved. There is a lack of information on the coupled setup (e.g. number of levels, atmospheric simulations, ...)

2) I have been surprised the authors do not validate their uncoupled simulations with respect to the coupled simulation using the EKE and the MKE. This is major weakness of the paper. The claim the setup is not the very same is true but it should not be as the setup of the uncoupled model should be comparable to the one of coupled model. For example a larger EKE in the coupled simulation with respect to the uncoupled simulation may lead to a larger $s_{w}$. It may cause a too large re-energization of the uncoupled simulation. The comparison between $s_{s t}$ from the coupled simulations and the uncoupled simulations are not good enough to validate the parameterization. One
Interactive comment
Printer-friendly version

Discussion paper 
could imagine the use of a constant $s_{w}$ (say of 0.3) would lead to similar discrepancies. The discrepancies should also be discussed and explained. EKE and MKE should be compared to the coupled simulation, that should be considered as the "true" when validating the parameterization.

3) As stated in the introduction, the current feedback to the atmosphere induces a dampening of the EKE. However, as shown by Renault et al. 2016b, this dampening is not only due to a reduction of the transfer of energy between the ocean and the atmosphere but to a negative WW'. It induces a sink of energy from the geostrophic currents to the atmosphere. Such a sink is present everywhere in the world ocean and, in particular, over the WBC such as the GS (e.g. Scott and Xu 2009, Renault et al., 2016c, Xu et al 2016). The current feedback to the atmosphere also induces a reduction (up to $30 \%$ ) of the mean WW (Scott and Xu 2009), that is even more pronounced when using an active ocean (up to 50\%, Renult et al, 2016c). This in turn causes a slow down of the mean circulation as shown by Pacanovski (1987), Luo et al (2005), and Renault at al. (2016c) for the North Atlantic Basin. A parameterization of the atmospheric response in an uncoupled simulation should be able to reproduce the current feedback induced changes of the transfer of energy between the ocean and the atmosphere. Again, the mean EKE and KE resulting from the uncoupled simulations should be validated and compared to the coupled simulations. This point should be introduced and discussed in the paper.

4) The statistics should be improved. For example, $s_{w}, s_{s t}$ estimates and correlations with the surface stability.

5) The study should better acknowledge what has been done in previous studies. e.g., the coupling coefficient $s_{w}$ and $s_{s t}$ have already been defined in the litterature. Some of the results shown in that paper are in agreement with previous studies (e.g. $s_{w}$ and $s_{s t}$ scatterplot) but this is not discussed or compared. What $s_{w}$ and $s_{s t}$ did you find for the US West coast?

Interactive comment
Printer-friendly version

Discussion paper 
Some specific comments:

Line 20: Hugues and Wilson (2008) and Scott and Xu (2009) do not use an active ocean. The reduction of mean WW induces a slow down of the current and then an additional reduction of the mean WW, see e.g. Renault 2016 for the NATL/GS. The reduction of EKE is not only explained by a reduction of the the wind energy transfer but to a negative geostrophic eddy wind work, see studies from Scott and Xu (2009), Renault et al. (2016bc), Xu et al (2016).

25: This is an interesting statement. Scott and Xu (2009), and Xu et al (2016) show the mean eddy wind work is also largely negative is those regions, which contradicts the Byrne et al (2016) results. It would have been interesting to check the mean eddy wind work in your simulation to address whether there is also a dampening of the EKE in that region or a re-energization as suggested by Byrne et al. (2016). Note Byrne et al (2016) use only 3 months of simulation, this could explain discrepancies with your own results.

page 2

\section{5}

The dampening of the EKE is explained by a negative geostrophic eddy wind work that allows a sink of energy from the geostrophic currents to the atmosphere (See Renault et al., 2016b). Such a sink of energy is overestimated when using an uncoupled ocean model. The current feedback induces large scale sink of energy from geostrophic currents to the atmosphere (Renault et al. 2016c Xu et al 2016) that explains the EKE dampening. This should be checked in the coupled and uncoupled simulations.

\section{Results}

Note Figure 1 is consistent with the results of Renault et al. (2016b) for the US West coast. It would have been interesting to compare your results to the literature.

page 8
OSD

Interactive

comment
Printer-friendly version

Discussion paper 
10: what about just extrapolating the values?

References:

OSD

Brodeau, Laurent, et al. "Climatologically Significant Effects of Some Approximations in the Bulk Parameterizations of Turbulent Air-Sea Fluxes." Journal of Physical Oceanography 47.1 (2017): 5-28.

Interactive

Luo, Jing-Jia, et al. "Reducing climatology bias in an ocean-atmosphere CGCM with improved coupling physics." Journal of climate 18.13 (2005): 2344-2360.

Pacanowski, R. C. "Effect of equatorial currents on surface stress." Journal of Physical Oceanography 17.6 (1987): 833-838.

Renault, Lionel, et al. "Modulation of wind work by oceanic current interaction with the atmosphere." Journal of Physical Oceanography 46.6 (2016b): 1685-1704.

Renault, Lionel, et al. "Control and Stabilization of the Gulf Stream by Oceanic Current Interaction with the Atmosphere." Journal of Physical Oceanography 46.11 (2016c): 3439-3453.

Scott, Robert B., and Yongsheng $\mathrm{Xu}$. "An update on the wind power input to the surface geostrophic flow of the World Ocean." Deep Sea Research Part I: Oceanographic Research Papers 56.3 (2009): 295-304.

Xu, Chi, Xiaoming Zhai, and XiaoâĂŘDong Shang. "Work done by atmospheric winds on mesoscale ocean eddies." Geophysical Research Letters 43.23 (2016).

Interactive comment on Ocean Sci. Discuss., doi:10.5194/os-2017-24, 2017. 